\title{
A wide area fault detection algorithm for transmission networks equipped with series compensation units
}

\author{
Mohamed A. Ebrahim', Fady Wadie ${ }^{2}$, Mousa A. Abd-Allah ${ }^{3}$ \\ ${ }^{1,3}$ Faculty of Engineering at Shoubra, Benha University, Egypt \\ ${ }^{2}$ Faculty of Engineering, Egyptian Russian University, Egypt
}

\begin{tabular}{l} 
Article Info \\
\hline Article history: \\
Received Sep 17, 2018 \\
Revised Dec 11, 2018 \\
Accepted Dec 28, 2018 \\
\hline
\end{tabular}

\section{Keywords:}

Adaptability

Backup protection

Phasor measurement units

Wide area back-up protection

\begin{abstract}
In this paper, a wide area back-up protection (WABP) algorithm is presented based on phasor measurement units (PMUs) measurements placed across the transmission network. The proposed algorithm computes a selected index named as positive sequence power index (PSPI) to detect faults in the network. Firstly, the network is subdivided into back-up protection zones. For each zone, the PSPI index is computed as absolute the value of the difference between the positive sequence sent and received phasor powers across the terminals of the zone. For each zone, the PSPI is compared to a predefined threshold to detect faults. One of the contributions in this paper is the usage of mathematical formulation to set the threshold values for each zone rather than experimental trials usually used in previous literature. In addition, the algorithm doesn't depend iterative solutions nor line parameters of the network as usually used in WABP schemes. These advantages enhance the degree of confidence in decisions of the algorithm and reduces computational burdens to trivial amount. The presented algorithm (Level-1) could be enhanced into (level-2) if PMUs are available on all buses and in such case, the faulty line could be detected directly in a single step. WSCC 9bus system and NE 39-bus systems were considered to accomplish this study. Severe cases for series compensated lines were taken into consideration including voltage or current inversion. Simulation results emphasis on algorithm's robustness and adaptability.
\end{abstract}

Copyright $@ 2019$ Institute of Advanced Engineering and Science. All rights reserved.

\section{Corresponding Author:}

Fady Wadie,

Faculty of Engineering, Egyptian Russian University, Badr city, Cairo, Egypt

Cairo-Suez road, Badr City, Cairo Governorate 2860-9117.

Email: engfadywadie@hotmail.com

\section{INTRODUCTION}

The increasing problems facing conventional backup protection schemes and their maloperation during highly stressed operating conditions had paved the way for finding alternatives [1]. Wide Area Backup Protection (WABP) schemes have provided alternative solutions for these drawbacks [2, 3]. The primary goal of WABP is to initiate a parallel backup protection algorithm that intervenes with the conventional backup protection of the transmission line to prevent its maloperation. The WABP capabilities were expanded by employing the Phasor Measurement Units (PMUs) especially for transmission lines protection $[4,5]$. The research community in this field has been divided into three categories. The first category depends on measuring the sequence electrical quantities [4, 6-9]. The second type relies on gathering and analyzing the information provided by the protective relays across the network [10-17]. The third category intended to use a lower amount of electrical measurements by using the pre-fault impedance matrix of the faulted zone which depends on network topology [18-21]. The main drawback of the first category is the requirement of PMUs to be placed on all buses which could be a challenging task in large electrical networks. The second category suffers from high degree of complexity and the usage of probabilistic approach in analysis which reduce the degree of certainty in the final decision and that is intolerable during stressed conditions. Finally, 
the third category tends to avoid the drawbacks of the first category by minimizing the number of PMUs but it suffers from using iterative solutions and depending on network line parameters which increases time delay and reduces decision confidence respectively.

This paper aims to propose a new WABP scheme that avoids the drawbacks of previous schemes even in the presence of series compensated transmission lines. The proposed algorithm aims to define the faulty line accurately even in most severe cases without requiring iterative solutions or line parameters. The proposed scheme is firstly presented as level-1 which is based on subdividing the network into monitored zones with PMUs placed only at their terminals. Level-1 firstly detects the faulty zone then the faulty line in a two-step algorithm. A second level (level-2) is presented in which the faulty line could detected in a single step but requires PMUs placement on all buses. Both protection algorithms were applied to Western System Coordinating Council (WSCC) 9-bus system and New England (NE) 39-bus system. The systems were simulated using MATLAB/Simulink software packages. The paper is organized as follows. Section 2 provides the main concept of the proposed alorithm. Section 3 presents Level-1 of the algorithm and its mathematical derivation and the simulation results. Section 4 repeats the same sequence for level-2. A comparative study is presented in Section 5. Finally; conclusions are derived in section 6.

\section{MAIN CONCEPT OF THE PROPOSED ALGORITHM}

The proposed algorithm requires PMUs to be placed at the terminals of the previously defined monitoring zones. The measurements acquired from the PMUs are used to evaluate the positive sequence power entering and leaving each zone which is equal to the power consumed by the loads within that zone. The absolute value of the difference of those powers is named as "Positive Sequence Power Index" or (PSPI). During normal operation, the PSPI is equal to the load power and the power lost across the lines within the zone. Since the line losses are negligible as compared to the system's power, the PSPI will be equal to load power within the zone. During normal operation, the load power is computed based on PMUs measurements and is used to update the threshold value used for fault detection. However, when a fault occurs within the protected zone, the PSPI will rise greatly above usual load power and above the threshold value showing that an additional element has been added into the zone that is consuming high power. Hence, fault is detected. Such claim will be mathematically proven and simulated to validate it.

\section{PROPOSED ALGORITHM (LEVEL-1)}

Level-1 of the proposed algorithm is executed in two steps; the first step uses PSPI to find the faulty zone. In step two, the positive sequence voltage measurements provided by the PMUs located at the terminal of the faulty zone are compared; the bus with the minimum positive sequence voltage will be the bus closest to the fault. Hence, the line connected to that bus is the faulty line. To ensure the validity of the proposed algorithm, firstly a mathematical analysis is presented in addition to the simulation results.

\subsection{Mathematical derivation}

A simple electrical network would be considered as shown in Figure 1. To apply PSPI as a monitoring tool, it should be capable of defining internal faults only as follows.

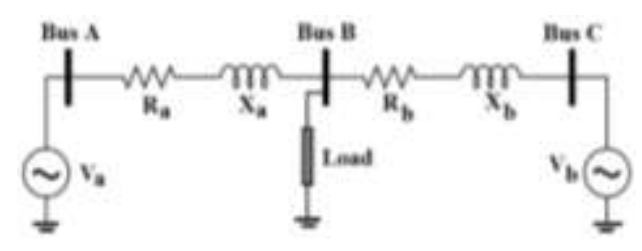

Figure 1. Simple electrical network

\subsubsection{No fault or external fault}

The PSPI was previously defined as the absolute difference of positive sequence powers across a zone. By considering the network presented network in Figure 1 as a general zone with PMUs placed on buses $\mathrm{A}$ and $\mathrm{C}$. The proof will firstly consider the PSPI across AB then it will be extended across AC. It could be noted that the positive sequence power received at bus B from A is equal to negative the value of the power sent from B to A. Therefore, the PSPI could be calculated for Line AB as follows: 


$$
\text { PSPI }=\left|\mathrm{P}_{\mathrm{AB} 1}+\mathrm{P}_{\mathrm{BA} 1}\right|
$$

Where: $\mathrm{P}_{\mathrm{AB} 1}, \mathrm{P}_{\mathrm{BA} 1}$ pos. seq. powers sent from bus $\mathrm{A}$ to $\mathrm{B}$ and bus $\mathrm{B}$ to $\mathrm{A}$ respectively. $\mathrm{P}_{\mathrm{AB} 1}$ and $\mathrm{P}_{\mathrm{BA} 1}$ could be calculated as follows:

$$
\mathrm{P}_{\mathrm{AB} 1}=\mathrm{V}_{\mathrm{A} 1} \mathrm{I}_{\mathrm{AB} 1} \cos \left(\varphi_{\mathrm{AB} 1}\right) \& \mathrm{P}_{\mathrm{BA} 1}=\mathrm{V}_{\mathrm{B} 1} \mathrm{I}_{\mathrm{BA} 1} \cos \left(\varphi_{\mathrm{BA} 1}\right)
$$

Where $V_{A 1}, V_{B 1}$ : pos. seq. voltages for bus $A$ and $B$ respectively. $I_{A B 1}, I_{B A 1}$ : pos. seq. currents flowing from bus $A$ to $B$, and from $B$ to $A$ respectively. $\cos \left(\varphi_{\mathrm{AB} 1}\right), \cos \left(\varphi_{\mathrm{BA} 1}\right):$ cosine the angle of the phase difference between $V_{A 1}$ and $I_{A B 1}, V_{B 1}$ and $I_{B A 1}$ respectively. Since all the current sent from bus $A$ is received by bus $B$ so it could be stated that

$$
\overrightarrow{\mathrm{I}}_{\mathrm{AB} 1}=-\overrightarrow{\mathrm{I}}_{\mathrm{BA} 1} \cdot \text { Hence: } \varphi_{\mathrm{I}_{\mathrm{BA} 1}}=\varphi_{\mathrm{I}_{\mathrm{AB} 1}}+180^{\circ}
$$

Where $\varphi_{\mathrm{I}_{\mathrm{BA} 1}}, \varphi_{\mathrm{I}_{\mathrm{AB} 1}}$ are the phase angles of currents $\mathrm{I}_{\mathrm{BA} 1}$ and $\mathrm{I}_{\mathrm{AB} 1}$ respectively. Then with the consideration of Equation (3), $\cos \left(\varphi_{\mathrm{BA} 1}\right)$ could be calculated as follows:

$$
\cos \left(\varphi_{\mathrm{BA} 1}\right)=\cos \left(\varphi_{\mathrm{VB} 1}-\varphi_{\mathrm{I}_{\mathrm{BA} 1}}\right)=-\cos \left(\varphi_{\mathrm{VB} 1}-\varphi_{\mathrm{I}_{\mathrm{AB} 1}}\right)
$$

Where $\varphi_{\mathrm{VB} 1}$ is the phase angle of $\mathrm{V}_{\mathrm{B} 1}$. Since the voltage drop across the line is relatively small as compared to the bus voltage. It could be assumed that both $V_{A 1}$ and $V_{B 1}$ are equal or that $\varphi_{\mathrm{VA} 1} \cong \varphi_{\mathrm{VB} 1}$ and $\left|\mathrm{V}_{\mathrm{A} 1}\right| \cong$ $\left|\mathrm{V}_{\mathrm{B} 1}\right|$. Equation (4) could be rewritten as follows:

$$
\cos \left(\varphi_{\mathrm{BA} 1}\right)=-\cos \left(\varphi_{\mathrm{VA} 1}-\varphi_{\mathrm{I}_{\mathrm{AB} 1}}\right)=-\cos \left(\varphi_{\mathrm{AB} 1}\right)
$$

By substituting the previous approximations where $\left|V_{A 1}\right| \cong\left|V_{B 1}\right|$, the magnitudes of $I_{A B 1}=I_{B A 1}$ and equation (5) into equation (2), we get: $\mathrm{P}_{\mathrm{BA} 1} \cong-\mathrm{V}_{\mathrm{A} 1} \mathrm{I}_{\mathrm{AB} 1} \cos \left(\varphi_{\mathrm{AB} 1}\right)$ or simply $\mathrm{P}_{\mathrm{BA} 1} \cong-\mathrm{P}_{\mathrm{AB} 1}$. Then PSPI $\cong$ $\left|\mathrm{P}_{\mathrm{AB} 1}-\mathrm{P}_{\mathrm{AB} 1}\right| \cong$ zero (6) Therefore, it could be concluded in case of no-fault condition; the PSPI for AB will be approximately equal to zero. The next step is to calculate the PSPI for the entire zone with PMUs only placed across bus A and C only:

$$
\text { PSPI }=\left|P_{\mathrm{AB} 1}+\mathrm{P}_{\mathrm{CB} 1}\right|
$$

Where $\mathrm{P}_{\mathrm{AB} 1}, \mathrm{P}_{\mathrm{CB} 1}$ is pos. seq. power sent from bus $\mathrm{A}$ to $\mathrm{B}$ and $\mathrm{C}$ to $\mathrm{B}$ respectively. For balanced systems, the positive sequence component of power is equal to the actual active power. In addition to that, the power sent from buses $\mathrm{A}$ and $\mathrm{C}$ will be equal to the power delivered to the different electrical loads and the power losses across the lines within the monitored zone.

$$
\left|\mathrm{P}_{\mathrm{AB} 1}+\mathrm{P}_{\mathrm{CB} 1}\right|=\left|\mathrm{P}_{\text {delivered to the zone }}\right|=\left|\mathrm{P}_{\mathrm{AB}}+\mathrm{P}_{\mathrm{BC}}+\mathrm{P}_{\text {load }}\right|
$$

where $\mathrm{P}_{\mathrm{AB}}, \mathrm{P}_{\mathrm{BC}}$ : are the active power losses of the lines $\mathrm{AB}$ and $\mathrm{BC}$ respectively. $\mathrm{P}_{\text {load }}$ : is the active load power consumed by the load connected to bus $\mathrm{B}$. As proven earlier, the power losses of the line $\mathrm{AB}$ and similarly BC are approximately zero for no-fault conditions. Then Equation (8) could be rewritten as follows:

$$
\text { PSPI }=\left|P_{\mathrm{AB} 1}+\mathrm{P}_{\mathrm{CB} 1}\right| \cong\left|\mathrm{P}_{\text {load }}\right|
$$

\subsubsection{Internal fault}

In this case for line $\mathrm{AB}$, the current sent from bus $\mathrm{A}$ will not be fully received by bus $\mathrm{B}$ due to the presence of a fault. In that case, there would be two currents flowing; one from bus A to the fault point and the second one from bus B to the fault point. So, for inductive loads with uncompensated lines:

$$
0^{\circ}<\varphi_{\mathrm{VA} 1}-\varphi_{\mathrm{I}_{\mathrm{AB} 1}}<90^{\circ} \& \quad 0^{\circ}<\varphi_{\mathrm{VB} 1}-\varphi_{\mathrm{I}_{\mathrm{BA} 1}}<90^{\circ}
$$

While for series compensated lines, the effect of the capacitive parts in the flowing currents could be taken into account by extending the range as flows:

$-90^{\circ}<\varphi_{\mathrm{VA} 1}-\varphi_{\mathrm{I}_{\mathrm{AB} 1}}<90^{\circ} \&-90^{\circ}<\varphi_{\mathrm{VB} 1}-\varphi_{\mathrm{I}_{\mathrm{BA} 1}}<90^{\circ}$ 
Thus both $\cos \left(\varphi_{B A 1}\right)$ and $\cos \left(\varphi_{A B 1}\right)$ will always have a positive value even with the existence of series compensation on the lines. Hence, it could be concluded that both $\mathrm{P}_{\mathrm{AB} 1}$ and $\mathrm{P}_{\mathrm{BA} 1}$ will always have positive values. Therefore, it could be concluded that:

$$
\text { PSPI }=\left|\mathrm{P}_{\mathrm{AB} 1}+\mathrm{P}_{\mathrm{BA} 1}\right|=\mathrm{P}_{\mathrm{AB} 1}+\mathrm{P}_{\mathrm{BA} 1}
$$

Since in case of faults, the currents rise to very high values compared to healthy operation, it is expected that both $\mathrm{P}_{\mathrm{AB} 1}$ and $\mathrm{P}_{\mathrm{AB} 1}$ will be of much higher value as compared to their values during healthy operation. Hence, the PSPI in case of fault will be of very high detectable value. To extend the proof across the entire network line $\mathrm{BC}$ is also considered by reapplying condition (11) to line BC as given in (13).

$$
-90^{\circ}<\varphi_{V B 1}-\varphi_{I_{B C 1}}<90^{\circ} \&-90^{\circ}<\varphi_{V C 1}-\varphi_{I_{C B 1}}<90^{\circ}
$$

Thus, both $\cos \left(\varphi_{\mathrm{AB} 1}\right)$ and $\cos \left(\varphi_{\mathrm{CB} 1}\right)$ will always have a positive value even with the existence of series compensation on the lines. Therefore, both $\mathrm{P}_{\mathrm{AB} 1}$ and $\mathrm{P}_{\mathrm{CB} 1}$ will always have positive values and the resulting power losses across line $\mathrm{AB}$ will be of significant value as it includes the power losses consumed by the fault. Hence, it could be concluded that:

$$
\text { PSPI }=\left|P_{\mathrm{AB} 1}+\mathrm{P}_{\mathrm{CB} 1}\right|=\mathrm{P}_{\mathrm{AB}}+\mathrm{P}_{\mathrm{CB}}+\mathrm{P}_{\text {load }}
$$

By comparing the PSPI value in case of internal fault as stated in (14) with the PSPI value in the event of no fault as indicated in (9), it is clear that in the case of fault, the PSPI will rise by a value equal to $\mathrm{P}_{\mathrm{AB}}+\mathrm{P}_{\mathrm{CB}}$ which will be of very high detctable value due to fault presence. The findings of this proof is applicable to other forms of sub-networks as long as only one unmonitored bus is allowed per zone.

\subsection{Threshold selection criterion}

Mathematical formulation for a line threshold value is firstly presented to be used in deriving a mathematical formula for the threshold value of monitored zones. The threshold value of a line which should be exceeded only in case of faulty conditions, could be given as:

$$
\text { Threshold }=\frac{\left|\mathrm{P}_{\mathrm{AB} 1 \mathrm{~h}}\right|+\left|\mathrm{P}_{\mathrm{BA} 1 \mathrm{~h}}\right|}{4}
$$

Where $\mathrm{P}_{\mathrm{AB} 1 \mathrm{~h}}, \mathrm{P}_{\mathrm{BA} 1 \mathrm{~h}}$ : are pos. seq. powers sent from buses $\mathrm{A}$ to $\mathrm{B}$ and $\mathrm{B}$ to $\mathrm{A}$ in healthy operating conditions respectively. Since the expected fault power for one line (stated in (12)) is for 3 phase faults, then the expected fault power for single-phase faults will be around one-third that value. Since the threshold value should be lower than all expected fault powers, it was selected as one-fourth of the value stated in (12) as given in (15). The threshold value is still much higher than of non-faulty condition power which is around zero. To calculate the threshold for the whole zone that would require the consideration of the active power consumed by the loads within the monitored zone. As proven before for no-load/ external fault condition, it is expected for the PSPI to be equal $\mathrm{P}_{\text {load }}$. While for internal fault condition, it is supposed to be much higher than $\mathrm{P}_{\text {load }}$ as expressed in (14). Hence, it is logical to shift the threshold setting value shown in (15) by an amount equal to $\mathrm{P}_{\text {load }}$ as shown in (16).

$$
\text { Threshold }=\mathrm{P}_{\mathrm{load}}+\frac{\left|\mathrm{P}_{\mathrm{AB} 1 \mathrm{~h}}\right|+\left|\mathrm{P}_{\mathrm{CB} 1 \mathrm{~h}}\right|}{4}
$$

By substituting Equation (9) into (16), we can rewrite the threshold as follows:

$$
\text { Threshold }=\operatorname{PSPI}_{\text {no-fault }}+\frac{\left|\mathrm{P}_{\mathrm{AB} 1 \mathrm{~h}}\right|+\left|\mathrm{P}_{\mathrm{CB} 1 \mathrm{~h}}\right|}{4}
$$

Where $\mathrm{P}_{\mathrm{AB} 1 \mathrm{~h}}, \mathrm{P}_{\mathrm{CB} 1 \mathrm{~h}}$ : are pos. seq. powers sent from bus $\mathrm{A}$ to $\mathrm{B}$ and from bus $\mathrm{C}$ to $\mathrm{B}$ in healthy operating conditions respectively. During no fault or external fault, the PSPI is approximately equal to the $\mathrm{P}_{\text {load }}$ and the calculated PSPI is far below the selected threshold. While for internal fault, the PSPI value is more than the threshold value by a significant margin. This threshold is updated on regular periodic basis to 
ensure that load power is continuously updated in order to cope with different variations of daily power loads. The algorithm could identify increase in load based on gradual increase in load power and incorporates that in threshold value in (17). Such case in simulated in case of load encroachment as it will be shown in the simulation section. While in case of faults, the sudden and large change in load is detected and defined as faults.

\subsection{Zone selection criterion}

When the faulty zone is identified using PSPI, only the pos. seq. voltages of the terminal buses are available from the PMUs located at the terminal buses of the monitored zone. If only one unmonitored bus is located in that zone and that bus is connected to all terminal buses, then it will be a common bus for all expected faulty lines. Therefore, it would only require the knowledge of the pos. seq. voltages at the terminal buses to define the faulty line. Thus, the conditions for zone selection are:

1. Only one unmonitored bus is allowed per zone.

2. The unmonitored bus must be connected to all monitored buses.

3. In case of series compensated lines or presence of a generator connected to the line, PMUs are placed on both terminals of the line.

Based on the previous conditions, it could be used to set a mathematical formula to define the buses required to be equipped with PMUs. Even more, an optimization algorithm is used to set the minimal number of PMUs required.

\subsubsection{Optimization of the number of the required PMUs:}

For any systems with " $n$ " buses, the main objective function is to minimize the value of the required PMUs named as " $\mathrm{x}$ "

$$
\mathrm{x}=\sum_{\mathrm{i}=0}^{\mathrm{n}} \mathrm{b}_{\mathrm{i}}
$$

Where " $\mathrm{i}$ " is the bus number, "b" is a binary variable with a value of zero in case that there is no PMU connected to bus or 1 in case a PMU is connected. The function is subject to constraint that for unmonitored bus " $k$ " where $\left(b_{k}=0\right)$, all the buses connected to it must be monitored to comply with the previous zone selection criterion. That can be expressed mathematically by defining a variable $\mathrm{y}_{\mathrm{k}}$ as follows:

$$
\mathrm{y}_{\mathrm{k}}=\sum_{\mathrm{i}=0}^{\mathrm{n}} \mathrm{L}_{\mathrm{ki}}\left(\mathrm{L}_{\mathrm{ki}}-\mathrm{b}_{\mathrm{i}}\right)=0 \quad \forall(k \neq i)
$$

Where $\mathrm{L}_{\mathrm{ki}}$ is a variable equals 1 in case a line exists between buses " $\mathrm{k}$ " and " $\mathrm{i}$ " otherwise equals to zero. To satisfy the constraint, $y_{k}$ must be equal to zero otherwise it means there will be a un-observable bus. Before applying the algorithm, all generator buses are equipped with PMUs. The results of the required number of PMUs are provided in Table 1. The optimization was carried out using nonlinear programming solver provided by GAMS environment software package.

Table 1. Number of the Required PMUs

\begin{tabular}{ccc}
\hline Test System & Number of PMUs & Buses equipped with PMUs \\
\hline WSCC 9-bus & 4 & $7,8,9,4$ \\
IEEE 14-bus & 8 & $1,2,4,5,6,9,10,13$ \\
NE 39-bus & 18 & $2,4,6,8,10,11,13,14,16,17,18,19,22,23,25,26,29,39$ \\
IEEE 57-bus & 32 & $1,2,3,4,6,8,9,11,12,13,14,19,21,22,24,27,29,30,31,33,34,36,38,39,41,45,47$, \\
4
\end{tabular}

The amount of PMUs could be furtherly reduced using optimal reduction techniques; however, this decrease may lead to more than one unmonitored bus within a zone which requires iterative solutions to identify the faulty bus resulting in deterioration in the WABP performance.

\subsection{Simulation results}

Two of the most commonly used benchmark systems were selected as testing systems; The WSCC 9-bus shown in Figure 2 with its data provided in [9] and the NE 39-bus system shown in Fiure 3 with its data from [22]. The series compensation level was set to $60 \%$ and $70 \%$ for 9-bus and NE 39-bus systems respectively. The PMUs were modeled with a reporting rate of 50 frames/second for $50 \mathrm{~Hz}$ system or simply 1 frame/cycle as defined in IEEE C37.118.1 [23]. The proposed algorithm was tested under stressed conditions including voltage/current inversion, various compensation levels, variable fault locations, stable 
power swing and load encroachment. Table 2 presents the monitored zone; the lines monitored within each area, and their respective thresholds were calculated by (17).

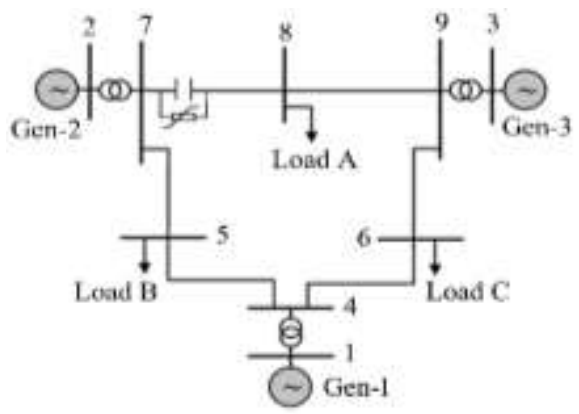

Figure 2. WSCC 9-bus system

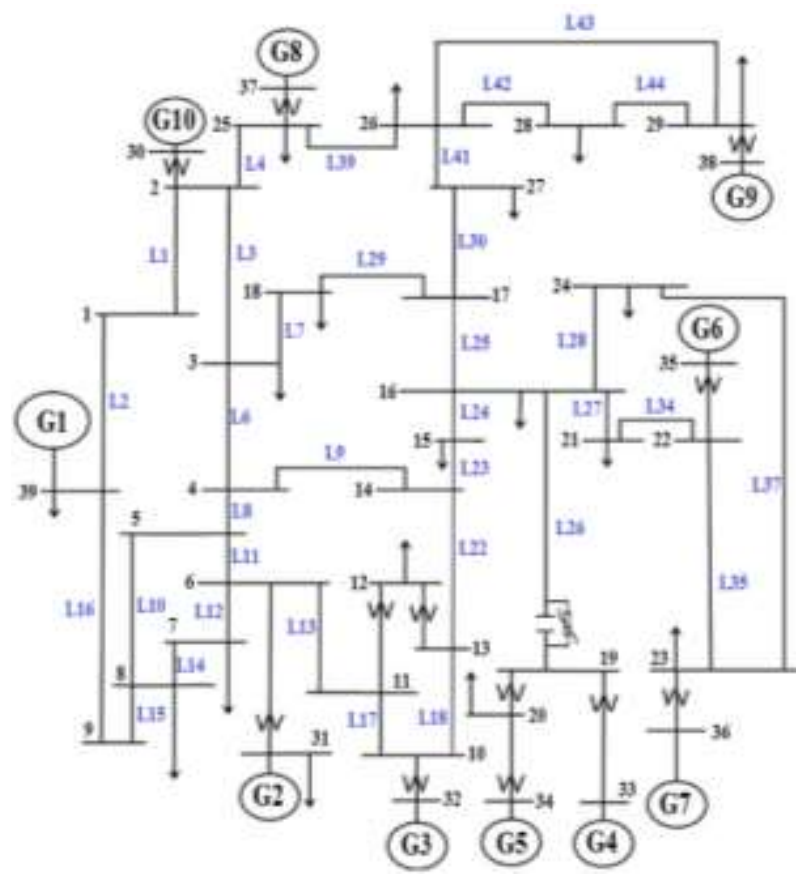

Figure 3. NE 39-bus system

Table 2. Proposed Algorithm (level-1) Monitoring Zones

\begin{tabular}{|c|c|c|c|c|c|c|c|c|c|c|c|}
\hline \multicolumn{12}{|c|}{ WSCC 9-bus system } \\
\hline Zone & \multicolumn{3}{|c|}{1} & \multicolumn{3}{|c|}{2} & \multicolumn{3}{|c|}{3} & \multicolumn{2}{|c|}{4} \\
\hline Monitored lines & \multicolumn{3}{|c|}{$7-8$} & \multicolumn{3}{|c|}{$8-9$} & \multicolumn{3}{|c|}{$9-6,6-4$} & \multicolumn{2}{|c|}{$7-5,5-4$} \\
\hline Threshold (p.u.) & \multirow{2}{*}{\multicolumn{3}{|c|}{0.2}} & \multicolumn{3}{|c|}{0.125} & \multirow{2}{*}{\multicolumn{3}{|c|}{0.5}} & \multirow{2}{*}{\multicolumn{2}{|c|}{0.67}} \\
\hline & & & & NE & 9-bus sy & & & & & & \\
\hline \multirow{2}{*}{$\begin{array}{l}\text { Zone } \\
\text { Monitored lines }\end{array}$} & 1 & 2 & 3 & 4 & 5 & 6 & 7 & 8 & 9 & 10 & 11 \\
\hline & 1,2 & $3,6,7$ & 13 & 4 & 29 & 9 & $\begin{array}{c}8,11 \\
10\end{array}$ & 12,14 & 17 & 16,15 & 22 \\
\hline \multirow{3}{*}{$\begin{array}{l}\text { Threshold (p.u.) } \\
\text { Zone } \\
\text { Monitored lines }\end{array}$} & 1 & 8 & 3.25 & 2 & 2 & 2 & 1.85 & 1.65 & 3.25 & 1 & 1.5 \\
\hline & 12 & 13 & 14 & 15 & 16 & 17 & 18 & 19 & 20 & 21 & 22 \\
\hline & $\begin{array}{l}24 \\
23\end{array}$ & 25 & 26 & 27,34 & 28,37 & $\begin{array}{l}30, \\
41\end{array}$ & 35 & 39 & 42,44 & 43 & 18 \\
\hline Threshold (p.u.) & 8 & 2.1 & 4 & 8 & 6.5 & 5 & 1 & 1 & 5 & 1.5 & 1.5 \\
\hline
\end{tabular}

\subsubsection{Voltage inversion and current inversion}

To provide adequate protection for series compensated transmission lines, it is mandatory to consider the accompanying voltage/current inversion phenomena. At both of these phenomena, the presence of series compensation leads to phase inversion of either voltage or currents behind the compensation point. Such inversions have been considered to be among the common problems facing conventional and WABP [4, $8,9]$. To simulate a voltage or current inversion, a fault is generally created on the series compensated line which is monitored as singular line for its critical effect. For voltage inversion, a single phase to ground fault (A-G) with the resistance of $1 \Omega$ was created in 0.03 seconds on line 7-8 of the 9-bus system at distance of 60 $\mathrm{km}$ from bus 7. The occurrence of voltage inversion is proven in Figure 4a. Only the PSPI of zone 1 exceeded its threshold of ( 0.2 p.u. $)$ as depicted in Figure $4 \mathrm{~b}$.

The current inversion was simulated by applying a 3-phase fault on line 26 of the NE 39-bus system at a distance from bus 19 equal to $30 \%$ of the line length. The PSPI of zone 14 exceeded its threshold as illustrated in Figure 5. Different fault types with fault resistance being varied from (1 to 6$) \Omega$ were tested for both 9-bus and 39-bus systems. Remarkably, the PSPI was capable of identifying the faulty line in all fault cases. 


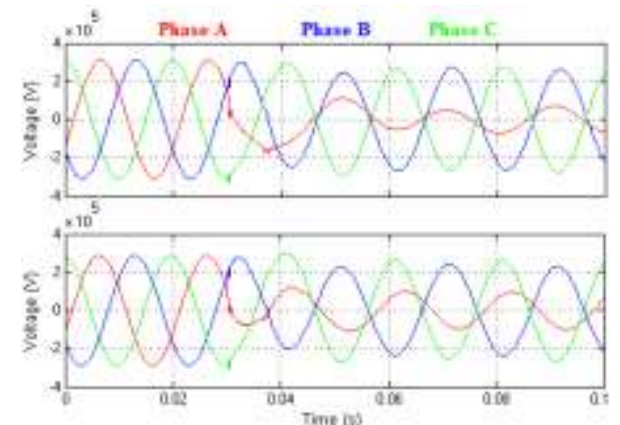

(a)

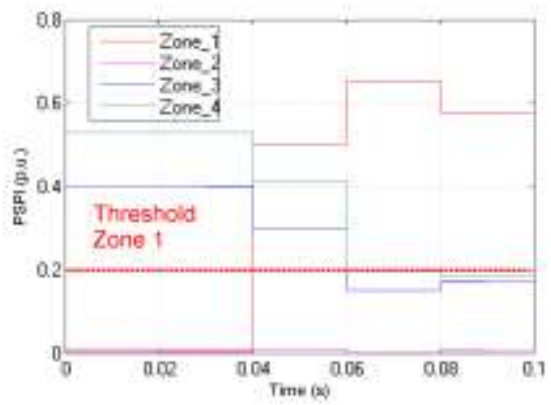

(b)

Figure 4. (a) Phase voltages at bus 7 (Top) and phase voltages behind series compensation unit (bottom) (b) PSPI (level-1) response on the 9-bus system in case of voltage inversion

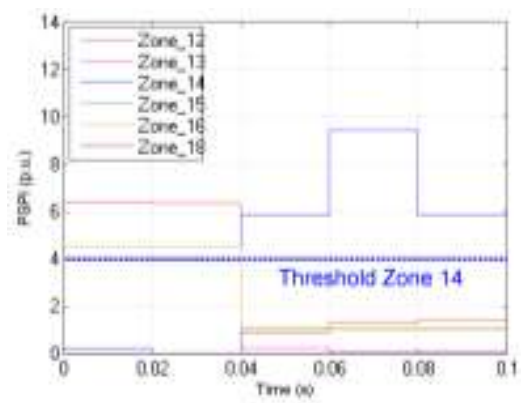

Figure 5. PSPI (level-1) response on the 39-bus in case of current inversion

\subsubsection{Different fault locations and compensation levels}

The effect of changing fault location from extreme ends of a monitored zone is examined. A three phase fault is created on line 7-5 of the 9-bus system at $30 \mathrm{~km}$ from bus 7 at $\mathrm{t}=0.02 \mathrm{~s}$. The PSPI response successfully identifies zone 4 as the faulty zone as illustrated in Figure $6 \mathrm{a}$. The positive sequence voltage of buses 4 and 7 (terminal buses of zone 4) are compared in Figure 6b. The comparison shows that bus 7 voltage suffered more significant reduction than that of bus 4 . Therefore, the faulty line is the one connected to bus 7 which is line 7-5. Also, the results showed that changing fault type, fault resistance or location did not affect the PSPI response which succeeded in identifying the faulty line in each time. The same conclusion was confirmed for both near and far ends while using the 39-bus system.

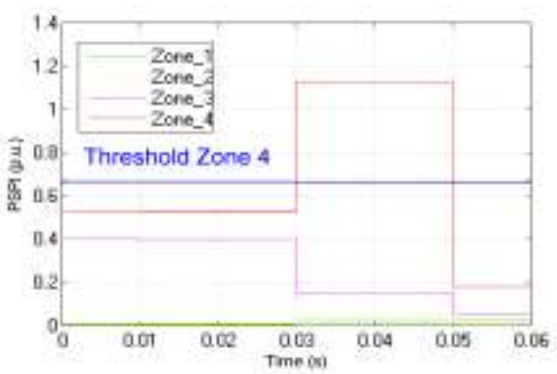

(a)

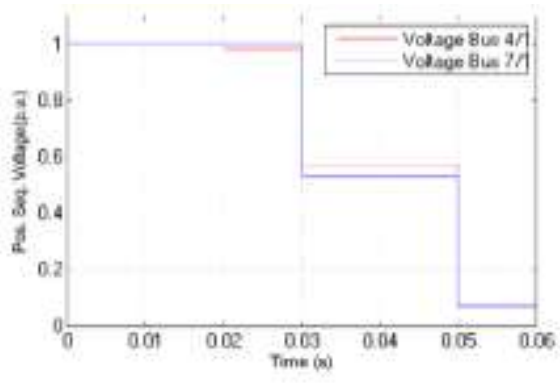

(b)

Figure 6. (a) PSPI (level-1) response for symmetrical fault (b) Pos. seq. bus-voltages

The compensation level was also varied from $70 \%$ to $40 \%$ with different fault locations and resistances for both 9-bus and 39-bus systems. A single phase to ground fault with the resistance of $100 \Omega$ was created on line 27 of the 39-bus system at a distance from bus 16 equal to $90 \%$ of the total line length. The previous conditions are meant to simulate low current faults with very high fault resistance located at far end of the line. The PSPI response and positive sequence bus voltages are shown in Figure $7 \mathrm{a}$ and $7 \mathrm{~b}$ respectively. The results show that PSPI response is independent of the series compensation level. 


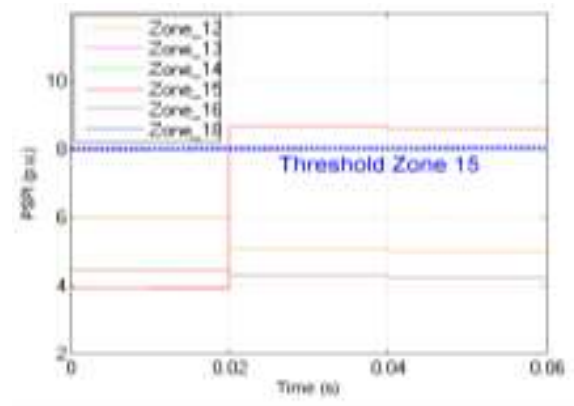

(a)

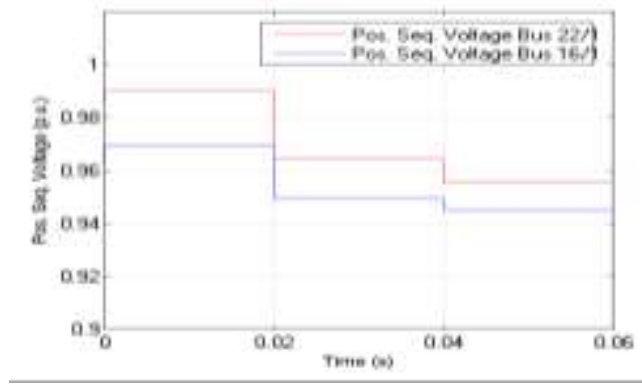

(b)

Figure 7. (a) PSPI (Level-1) Response on 39-bus System for Unsymmetrical Fault (b) Pos. Seq. Voltage

\subsubsection{Load encroachment and stable power swing}

To simulate load encroachment, a case similar to that presented in [9] was carried out on the 9-bus system by increasing the load at bus 8 gradually without exceeding the North American Electric Reliability Corporation (NERC) "extreme" emergency loading limit [9]. The increase in load would result for the impedance seen by quadrilateral distance relay located at bus 7 to enter its zone 3 as shown in Figure 8. That would lead to unnecessary tripping of the circuit breakers connecting line 8-9.

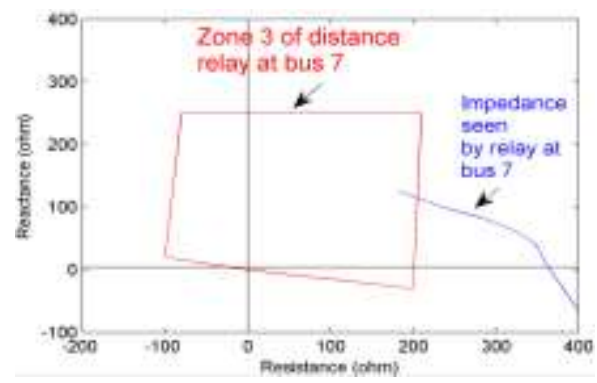

Figure 8. R-X plane showing the path of impedance seen by relay at bus 7 during load encroachment

On the other hand, the proposed algorithm doesn't pick up any fault signals as the PSPI of the zones did not exceed their threshold values as depicted in Figure 9a. For stable power swing, the case introduced in [9] was recreated in which a three-phase fault was presented on the midpoint of line 57 at $0.03 \mathrm{~s}$, it sustained for $0.03 \mathrm{~s}$ and was cleared at $0.06 \mathrm{~s}$. The sudden removal of line 57 from the system arises a stable power swing into the system. The response of the algorithm shown in Figure $9 \mathrm{~b}$ illustrates that only zone 4 exceeded its threshold at $0.04 \mathrm{~s}$. At $0.06 \mathrm{~s}$, the fault was cleared, and yet no PSPI of any line exceeded its threshold. Hence, the proposed algorithm wasn't hit by the power swing.

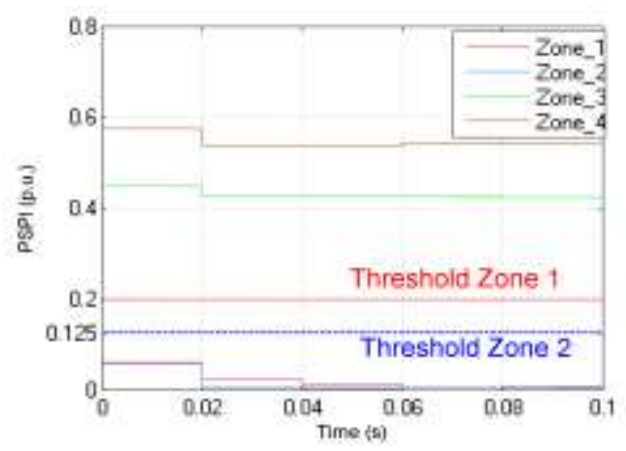

(a)

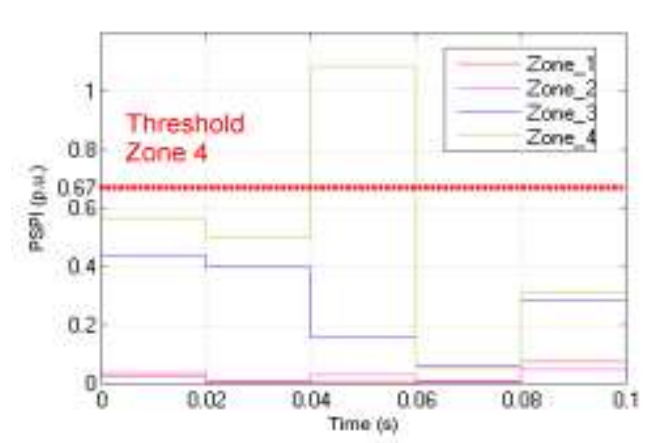

(b)

Figure 9. PSPI (level-1) response on 9-bus system for (a) Load encroachment (b) Power swing 


\section{PROPOSED ALGORITHM (LEVEL -2)}

The first level of the proposed algorithm (level-1) had showed great ability in detecting faults regardless of stressed conditions. However, the detection process is done in two steps. The number of steps could be reduced into a single step if PMUs are available on all buses. It should be noted that the requirement for applying level-2 could not be applicable for networks with large number of buses. Hence, level-1 of the proposed algorithm is considered the more reliable solution for today's networks while level-2 is only allowable for small scale networks. For level-2, the PMUs were placed on buses 4, 5, 6, 7, 8 and 9 to monitor all the lines of the 9-bus system. For the 39-bus system, PMUs were placed on buses 1 to 11, 13 to 19, 21 to 29 and 39 to monitor all 34 lines of the system. The mathematical formula of this level was previously introduced in (7), (12) for no-fault/external fault and internal fault respectively. The threshold values calculated using equation (15) for each line are listed in Table 3. The Robustness of the proposed level-2 PSPI algorithm against various types of faults at different locations, voltage and current inversions, load encorachement and power swings is inhertely verified within level-1 simulation results. As, level-1 succeeds with PMUs on zone terminals, level-2 with PMUs on line terminal will confidently provide the same results. Such claim was verified by recreating the previous simulation cases for level-2 and the results confirmed that conclusion.

Table 3. Proposed Algorithm (Level-2) Threshold Values

\begin{tabular}{|c|c|c|c|c|c|c|c|c|c|c|c|c|}
\hline \multicolumn{13}{|c|}{ WSCC 9-bus system } \\
\hline Line & \multicolumn{2}{|c|}{78} & \multicolumn{2}{|c|}{89} & \multicolumn{2}{|c|}{69} & \multicolumn{2}{|c|}{64} & \multicolumn{2}{|c|}{45} & \multicolumn{2}{|c|}{57} \\
\hline Threshold (p.u.) & \multicolumn{2}{|c|}{0.2} & & & \multicolumn{2}{|c|}{0.1} & \multicolumn{2}{|c|}{0.15} & \multicolumn{2}{|c|}{0.2} & \multicolumn{2}{|c|}{0.1} \\
\hline & & & & & NE 39-! & syster & & & & & & \\
\hline Line & 1 & 2 & 3 & 4 & 6 & 7 & 8 & 9 & 10 & 11 & 12 & 13 \\
\hline Threshold(p.u.) & 1 & 11 & 3 & 2 & 1.5 & 12 & 1 & 2 & 1.6 & 1.8 & 1.6 & 3.5 \\
\hline Line & 14 & 15 & 16 & 17 & 18 & 22 & 23 & 24 & 25 & 26 & 27 & 28 \\
\hline Threshold(p.u.) & 1.6 & 1 & 1 & 3.7 & 1.5 & 1.5 & 1 & 2.8 & 2.1 & 4 & 2.7 & 1 \\
\hline Line & 29 & 30 & 34 & 35 & 37 & 39 & 41 & 42 & 43 & 44 & & \\
\hline Threshold(p.u.) & 22 & 11 & 4.7 & 1 & 2.7 & 1 & 2 & 1.1 & 1.5 & 2.6 & & \\
\hline
\end{tabular}

\section{COMPARATIVE STUDY}

To express the contribution of the proposed algorithm, a comparative study is presented in two main subsections; comparative study concerning conventional current differential protection and concerning previous WABP schemes.

\subsection{Comparative study concerning differential protection}

It is essential to evaluate the proposed algorithm based on its primary task which intends to provide a wide area based backup protection that doesn't replace conventional backup protection but rather prevents it from maloperation. Such a trend was previously adopted by $[4,8,9]$. On such basis, the PSPI-based schemes could be compared to the current differential protection (DP) including three main cases arise as follows:

a. High resistance faults: it was stated in $[9,24]$ that DP finds limitations with high resistance faults. To further demonstrate that point, a specific case was simulated in [9] in which the DP failed in detecting the fault. That case was recreated by creating an A-G fault of (300 $\Omega$ ) fault resistance on line 7-8 of the 9-bus system at the far end from bus 7. That case was recreated to test the proposed algorithm (level-2) as shown in Figure 10. The figure clearly demonstrates that the PSPI successfully identified the faulty line even though that DP failed for such a very low current fault. A similar case is used for PSPI (level-1) in Figure 7. In other words, phasor power signal is more reliable than current signal as a fault detecting tool.

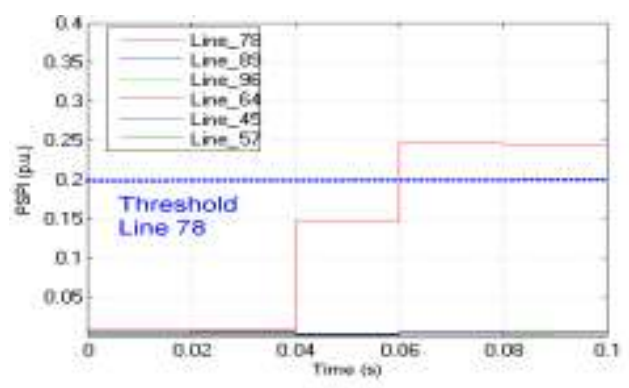

Figure 10. PSPI (level-2) response for high resistance fault located at the far end of line 
b. Current inversion: For any protective scheme dealing with series compensated transmission lines, current inversion represents one of the main issues that must be handled. The current DP may fail in current inversion situations as reported in [25, 26]. On the other hand, the proposed PSPI-based algorithm successfully overcame such a problem as presented in current/voltage inversion subsections of this article.

c. Synchronization error: for any differential based scheme, synchronization between the two quantities to be differentiated is a vital issue. Such error due to synchronization malfunction could be faced by current DP [27]. While in recent WABP schemes (including proposed algorithm) a GPS-based unique time stamp is given for each measurement with accuracy up to $1 \mu \mathrm{s}$ according to IEEE standard [28]. Such a property significantly reduces the possibility of synchronization error in the proposed schemes.

The success of PSPI in the previous cases could be attributed to the selection of phasor power signal rather than current signal. This could be furtherly explained as the power does not only depend on the magnitudes of voltage and currents, but also on the phase shift between voltage and current. Such shift increases dramatically in case of fault incidents as current reverses its direction towards the fault. For such reason, PSPI was capable of identifying faults even for extremely low fault currents as in Figure 7 and Figure 10 where current signal failed as reported in [9]. In addition to that, PMUs are increasingly deployed in modern-day networks [29] as part of many applications including but not limited to: state estimation, power system monitoring and control, and stability studies [30]. Hence, the proposed scheme does not add any additional requirements to modern day systems but rather employs the capabilities of PMUs to provide a more reliable backup protection.

\subsection{Comparative study with respect to previous WABP schemes}

The work presented is an extension of the works presented in [4, 8, 9]. Therefore, its merits upon its predecessors must be presented. A comparison between the proposed algorithm and its predecessors is provided in Table 4. Table 4 shows that so far scheme presented in [9] was more practical than those presented in [8, 4]. Both levels of the proposed algorithm show the same pros as that in [9] but they supersede it by relying on a simple mathematical difference operation, there's no possibility for any mathematical errors. While the scheme in [9] calculates the integrated impedance by dividing the bus voltages by the difference of the sent and received currents, which could lead to a possible division by zero error as in case of no fault the sent and received currents are almost equal. As an example of such case is the case shown in Figure 6. The results of that case while using the scheme in [9] is presented in Figure 11.

Table 4. Comparison of Proposed Algorithm (Levels $1 \&$ 2) and Other Schemes

\begin{tabular}{lcccc}
\hline & Level 1 & Level 2 & Scheme in [9] & Scheme in [8] \\
\hline $\begin{array}{l}\text { Operation for voltage/ current } \\
\text { inversion }\end{array}$ & Succeeds & Succeeds & Succeeds & Succeeds except for case reported in [9] \\
Required data & Pos. seq. voltage & Pos. seq. voltage & Pos. seq. V and & Pos., Neg. and zero sequence voltage \\
and currents & and currents & currents & and currents \\
Required PMUs for "N"-buses & $<0.65 \mathrm{~N}$ & $\mathrm{~N}$ & one & two \\
No. of steps & two & one & low & medium \\
Time delay & medium & low & Possible & None \\
Math. errors & None & None & & two \\
\hline
\end{tabular}

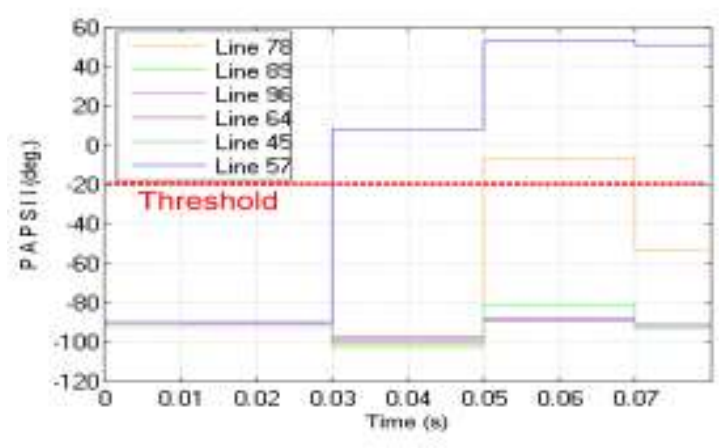

Figure 11. PAPSII response for symmetrical fault at $30 \mathrm{~km}$ from bus 7 on line 57 
The figure shows the phase angle of the positive sequence integrated impedance (PAPSII) of the system lines. As the fault is on line 57, it is expected that only line 57 exceeds the threshold. However, at the instant of $0.05 \mathrm{~s}$, the PAPSII of line 78 exceeds the threshold caused by a division by zero mathematical error. Such error would cause unnecessary tripping of line 78 or even infinite loop in the computing process which might lead to the system crash. Based on the previous study the main contribution of PSPI could be concluded:

a. The proposed algorithm succeeds in using low number of PMUs without requiring to use iterative solutions as in [18] which causes additional time delay in detection and an error margin in the decision. Also, it doesn't depend on line parameters as in [19] which could be subject to error depending on line parameter's accuracy during calculation. In other words, the PSPI succeeds in reducing the number of PMUs without compromising its reliability.

b. The algorithm computes its threshold based on a solid mathematical formula rather than experimental trials [ 7, 8, 9 and 19]. Such selection enhances the scheme reliability.

c. The proposed algorithm is highly reliable for series compensated lines protection even in stressed conditions unlike conventional DP and other WABP schemes [7,8] since it does not maloperate for voltage/current inversions, load encroachment or power swings.

d. The proposed algorithm is highly reliable and selective as a back-up protection scheme as it does not maloperate for most critical fault cases such as high fault impedance.

e. The algorithm is very simple in application, easy to set-up and requires trivial computational burdens.

f. The proposed algorithm is more reliable than DP as it employs GPS-based time stamps to ensure continuous synchronization between measured data eliminating the possibility of synchronization errors.

\section{CONCLUSION}

The proposed positive sequence power index (PSPI) with its two levels of application is carried out to overcome conventional backup protection problems and current WABP problem's. The proposed algorithm (both levels) was tested upon the WSCC 9-bus system and the NE 39-bus system using MATLAB as the simulation platform. The proposed algorithm is capable of providing adequate protection for series compensated transmission lines. As proven by simulation results and mathematical derivation, the PSPI is capable of identifying fault correctly even in severe cases of voltage or current inversion, different compensation levels, different fault types, locations and fault resistance. Comparing the proposed PSPI-based WABP algorithm to its predecessor schemes has proved the superiority of the proposed algorithm in capturing a simple index free of possible mathematical errors with flexibility in the required number of PMUs.

The proposed algorithm showed great adaptability to network requirements as level-1 could be used for large networks by using limited numbers of PMUs without requiring line data or using iterative solutions. For smaller networks, level-2 could be deployed if PMUs are available on all buses allowing detection of faulty lines in a single step.

\section{REFERENCES}

[1] S. H. Horowitz and A. G. Phadke, "Third zone revisited," in IEEE Transactions on Power Delivery, vol. 21, no. 1, pp. 23-29, Jan. 2006.

[2] J. Xiao, F. Wen, C. y. Chung and K. p. Wong, "Wide-Area Protection and its Applications - A Bibliographical Survey," 2006 IEEE PES Power Systems Conference and Exposition, Atlanta, GA, 2006, pp. 1388-1397.

[3] J. Bertsch, C. Carnal, D. Karlson, J. McDaniel and Khoi Vu, "Wide-Area Protection and Power System Utilization," in Proceedings of the IEEE, vol. 93, no. 5, pp. 997-1003, May 2005.

[4] M. M. Eissa, M. E. Masoud and M. M. M. Elanwar, "A Novel Back Up Wide Area Protection Technique for Power Transmission Grids Using Phasor Measurement Unit," in IEEE Transactions on Power Delivery, vol. 25, no. 1, pp. 270-278, Jan. 2010.

[5] M. M. Eissa, "New principle for transmission line protection using phase portrait plane," in IET Generation, Transmission \& Distribution, vol. 3, no. 1, pp. 49-56, January 2009.

[6] S. N. Muneshwar, R. P. Hasabet, P. Kose and A. A. Bhole, "A new adaptive PMU based protection scheme for interconnected transmission network system," 2014 International Conference on Circuits, Power and Computing Technologies [ICCPCT-2014], Nagercoil, 2014, pp. 179-183.

[7] Z. He, Z. Zhang, W. Chen, O. P. Malik and X. Yin, "Wide-Area Backup Protection Algorithm Based on Fault Component Voltage Distribution," in IEEE Transactions on Power Delivery, vol. 26, no. 4, pp. 2752-2760, Oct. 2011.

[8] P. K. Nayak, A. K. Pradhan and P. Bajpai, "Wide-Area Measurement-Based Backup Protection for Power Network With Series Compensation," in IEEE Transactions on Power Delivery, vol. 29, no. 4, pp. 1970-1977, Aug. 2014. 
[9] M. K. Jena, S. R. Samantaray and B. K. Panigrahi, "A New Wide-Area Backup Protection Scheme for SeriesCompensated Transmission System," in IEEE Systems Journal, vol. 11, no. 3, pp. 1877-1887, Sept. 2017.

[10] J. C. Tan, P. A. Crossley, D. Kirschen, J. Goody and J. A. Downes, "An expert system for the back-up protection of a transmission network," in IEEE Transactions on Power Delivery, vol. 15, no. 2, pp. 508-514, April 2000.

[11] J. C. Tan, P. A. Crossley, P. G. McLaren, P. F. Gale, I. Hall and J. Farrell, "Application of a wide area backup protection expert system to prevent cascading outages," in IEEE Transactions on Power Delivery, vol. 17, no. 2, pp. 375-380, April 2002.

[12] J. C. Tan, P. A. Crossley, P. G. McLaren, I. Hall, J. Farrell and P. Gale, "Sequential tripping strategy for a transmission network back-up protection expert system," in IEEE Transactions on Power Delivery, vol. 17, no. 1, pp. 68-74, Jan. 2002.

[13] Z. Li, X. Yin, Z. Zhang and Z. He, "Wide-Area Protection Fault Identification Algorithm Based on MultiInformation Fusion," in IEEE Transactions on Power Delivery, vol. 28, no. 3, pp. 1348-1355, July 2013.

[14] J. Ma, C. Liu and J. S. Thorp, "A Wide-Area Backup Protection Algorithm Based on Distance Protection Fitting Factor," in IEEE Transactions on Power Delivery, vol. 31, no. 5, pp. 2196-2205, Oct. 2016.

[15] Yang Zengli, Shi Dongyuan, and Duan Xianzhong, "Wide-area protection system based on direction comparison principle," Proceedings of the Chinese Society of Electrical Engineering, vol.28, no. 22, pp. 87-93, 2008.

[16] Y. Wang, X. Yin and D. You, "Agent-based wide area protection with high fault tolerance," Proceedings of the 2010 International Conference on Modelling, Identification and Control, Okayama, 2010, pp. 739-744.

[17] M. Chen, H. Wang, S. Shen and B. He, "Research on a Distance Relay-Based Wide-Area Backup Protection Algorithm for Transmission Lines," in IEEE Transactions on Power Delivery, vol. 32, no. 1, pp. 97-105, Feb. 2017.

[18] M. K. Neyestanaki and A. M. Ranjbar, "An Adaptive PMU-Based Wide Area Backup Protection Scheme for Power Transmission Lines," in IEEE Transactions on Smart Grid, vol. 6, no. 3, pp. 1550-1559, May 2015.

[19] J. Zare, F. Aminifar and M. Sanaye-Pasand, "Synchrophasor-Based Wide-Area Backup Protection Scheme with Data Requirement Analysis," in IEEE Transactions on Power Delivery, vol. 30, no. 3, pp. 1410-1419, June 2015.

[20] J. Ma, J. Li, J. S. Thorp, A. J. Arana, Q. Yang and A. G. Phadke, "A Fault Steady State Component-Based Wide Area Backup Protection Algorithm," in IEEE Transactions on Smart Grid, vol. 2, no. 3, pp. 468-475, Sept. 2011.

[21] P. Kundu and A. K. Pradhan, "Online identification of protection element failure using wide area measurements," in IET Generation, Transmission \& Distribution, vol. 9, no. 2, pp. 115-123, 2912015.

[22] A. Pai, "Energy function analysis for power system stability," Springer Science \& Business Media, 2012.

[23] IEEE Standard for Synchrophasor Measurements for Power Systems," in IEEE Std C37.118.1-2011 (Revision of IEEE Std C37.118-2005), vol., no., pp.1-61, 28 Dec. 2011.

[24] H. K. Zadeh and Z. Li, "Phasor measurement unit based transmission line protection scheme design," Electric Power Systems Research, vol. 81, no. 2, pp. 421-429, February 2011.

[25] H. J. Altuve, J. B. Mooney and G. E. Alexander, "Advances in series-compensated line protection," $200962 \mathrm{nd}$ Annual Conference for Protective Relay Engineers, Austin, TX, 2009, pp. 263-275.

[26] Y. Liu, A. P. S. Meliopoulos, R. Fan, L. Sun and Z. Tan, "Dynamic State Estimation Based Protection on Series Compensated Transmission Lines," in IEEE Transactions on Power Delivery, vol. 32, no. 5, pp. 2199-2209, Oct. 2017.

[27] S. Dambhare, S. A. Soman and M. C. Chandorkar, "Adaptive Current Differential Protection Schemes for Transmission-Line Protection," in IEEE Transactions on Power Delivery, vol. 24, no. 4, pp. 1832-1841, Oct. 2009.

[28] IEEE Standard Profile for Use of IEEE 1588 Precision Time Protocol in Power System Applications," in IEEE Std C37.238-2017 (Revision of IEEE Std C37.238-2011), vol., no., pp.1-42, 19 June 2017

[29] T. Rauhala, K. Saarinen, M. Latvala, M. Laasonen and M. Uusitalo, "Applications of phasor measurement units and wide-area measurement system in Finland," 2011 IEEE Trondheim PowerTech, Trondheim, 2011, pp. 1-8.

[30] J. De La Ree, V. Centeno, J. S. Thorp and A. G. Phadke, "Synchronized Phasor Measurement Applications in Power Systems," in IEEE Transactions on Smart Grid, vol. 1, no. 1, pp. 20-27, June 2010. 